\title{
Eyes on the prize
}

\author{
The 2014 Kavli Prize in Nanoscience has been awarded to Thomas Ebbesen, Stefan Hell and John Pendry \\ for their contributions to the field of nano-optics.
}

The Kavli Foundation and the Norwegian Academy of Science and Letters award three Kavli prizes - one in astrophysics, one in nanoscience and one in neuroscience every two years. This year the Kavli Prize in Nanoscience has been awarded to three distinguished scientists in recognition of advances in nano-optics that have challenged established beliefs on the behaviour of light interacting with subwavelength objects, and have helped overcome the diffraction limit in optical imaging. Thomas Ebbesen discovered extraordinary optical transmission, in which light transmits through subwavelength holes; Stefan Hell developed the technique called stimulated emission depletion microscopy, which can achieve imaging with a resolution down to tens of nanometres; and John Pendry invented metamaterials that have a negative refractive index, and, based on these materials, proposed the concept of a 'perfect lens'.

In its short history, the Kavli Prize has often provided a seemingly distinct flavour compared with other high-profile awards, such as the Nobel Prize, by rewarding contributions that go beyond a single remarkable discovery. In 2012, for example, the prize was awarded to Mildred Dresselhaus in recognition of her decades of pioneering work on carbon materials. The importance of the winner's research career, and the ways in which it has impacted different research areas over

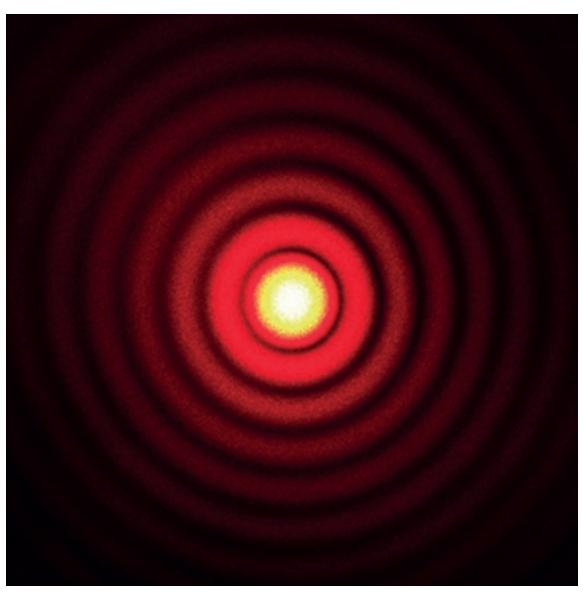

time, is thus emphasized, providing a fuller profile of the recipients. This, combined with the fact that prizes are awarded in welldefined areas of research, perhaps makes the Kavli Prize less controversial than others.

Whether or not the choice of recipients is a popular one, it is perhaps instructive to reflect on what the impact of these highprofile prizes is, or should be. The prizes are certainly effective at bringing a wider fame, almost overnight, to the winners. The prospects of worldwide recognition may also inspire young, and not-so-young, scientists to strive for excellence. Furthermore, by reaching to the general public, the Kavli and other prizes help raise the wider profile of science. As most research output is publicly funded, it is important that scientific work is understood and appreciated, and public outreach is probably one of the most important roles these prizes can play.

The prizes can, however, also create issues within the scientific community. They are awarded by committees, usually following the recommendation of academics and scientific organizations. Ultimately, though, the decisions rest with the few members of the committee. And yet the consequences of their choice are widespread: a research area can receive considerable attention, which can potentially lead to hype (graphene being an obvious example from recent years), and funding allocation can be skewed towards a particular field, at the expense of others. Of course, this may also happen, independently of prizes, as fields come in and out of fashion. And what about the financial reward that can accompany these prizes? It could be argued that it would be better to devolve this money to research centres, particularly when the prize is awarded to scientists who are no longer active in research.

It is clear though that these prizes can have a positive influence on science and on the public perception of science. The prizes will, of course, also have an influence on the winners themselves, and for such worthy recipients as Ebbesen, Hell and Pendry they may even drive the researchers on to further important scientific discoveries.

\section{Join us in the classroom}

\author{
We are launching a page dedicated to education.
}

In November 2013, we published a number of articles about nanoscience education. We asked scientists involved in education to discuss the challenges of teaching such a multidisciplinary topic, and we asked students to comment on their own education experience. We covered several aspects, but felt that the topic deserved more attention in the journal. From this issue we will be publishing each month a page titled 'In the Classroom' - dedicated to nanoscience education.
The 'classroom' we have in mind here is a generic place of learning. We want to provide a venue for scientists involved in education to discuss their ideas and for students to share their learning experiences. We would also like to use this space, on occasion, to provide our own perspective.

For the first article, we asked Doug Natelson of Rice University in the US to share his thoughts on nanoscience education based on his experience as a scientist and teacher; and he provides a series of interesting pointers. No doubt some may disagree with his views on the topic, but we hope these points will, at least, be the start of further debate on the subject.

Although we intend to commission most of the articles, if you are interested in contributing to this section, please send us yourideas at naturenano@nature.com. While we won't be able to publish everything we receive, we'll do our best to accommodate the most original views and perspectives. 